\title{
ANALYSIS AND NUMERICAL SOLUTION OF A RIEMANN-LIOUVILLE FRACTIONAL DERIVATIVE TWO-POINT BOUNDARY VALUE PROBLEM
}

\author{
NATALIA KOPTEVA ${ }^{\ddagger}$ AND MARTIN STYNES ${ }^{\dagger}$
}

\begin{abstract}
A two-point boundary value problem is considered on the interval $[0,1]$, where the leading term in the differential operator is a Riemann-Liouville fractional derivative of order $2-\delta$ with $0<\delta<1$. It is shown that any solution of such a problem can be expressed in terms of solutions to two associated weakly singular Volterra integral equations of the second kind. As a consequence, existence and uniqueness of a solution to the boundary value problem are proved, the structure of this solution is elucidated, and sharp bounds on its derivatives (in terms of the parameter $\delta$ ) are derived. These results show that in general the first-order derivative of the solution will blow up at $x=0$, so accurate numerical solution of this class of problems is not straightforward. The reformulation of the boundary problem in terms of Volterra integral equations enables the construction of two distinct numerical methods for its solution, both based on piecewise-polynomial collocation. Convergence rates for these methods are proved and numerical results are presented to demonstrate their performance.
\end{abstract}

Key words. Fractional differential equation, Riemann-Liouville fractional derivative, boundary value problem, weakly singular Volterra integral equation, collocation method.

AMS subject classifications. MSC (2010) Classification: Primary 65L10, Secondary 34B08, 65L60, 65L70.

1. Introduction. At present there is much active research into the design and analysis of numerical methods for differential equations containing fractional-order derivative because these derivatives are useful in modelling certain physical processes; see the discussion and references in [5]. In particular, numerical methods for two-point boundary value problems involving Riemann-Liouville derivatives have been examined in many papers. Despite this high level of activity, existence/uniqueness/regularity results for this class of problems have been confined to problems where the differential operator does not include a convective term (see $[4,5]$ and their references).

To analyse rigorously the convergence of any numerical method for RiemannLiouville fractional-derivative boundary value problems, one needs information about the existence, uniqueness and regularity of the solution. The pointwise regularity of solutions to Riemann-Liouville boundary value problems that include a convective term is still an open question, and as a consequence there are currently no rigorous convergence results for finite difference or collocation methods for this class of problems.

In the present paper we make two contributions to filling this gap in the literature. We prove existence, uniqueness and regularity (in a pointwise sense) for a class of Riemann-Liouville two-point boundary problems that permits convective terms, and present an efficient collocation method for their solution for which we obtain rigorous error bounds.

The structure of the paper is as follows. Section 1 presents the fractionalderivative two-point boundary value problem that we study. In Section 2 it is shown that the solution of this problem is equivalent to the solution of a pair of weakly

\footnotetext{
${ }^{\ddagger}$ Department of Mathematics and Statistics, University of Limerick, Ireland; natalia.kopteva@ul.ie

${ }^{\dagger}$ Applied and Computational Mathematics Division, Beijing Computational Science Research Center, China; m.stynes@csrc.ac.cn
} 
singular Volterra integral equations of the second kind. This enables us to prove existence and uniqueness of a solution to the original boundary value problem. Then in Section 3 we construct and analyse two numerical methods for solving the boundary value problem that are based on Volterra integral equation reformulations of the problem.

Notation. We use the standard notation $C^{k}(I)$ to denote the space of real-valued functions whose derivatives up to order $k$ are continuous on an interval $I$, and write $C(I)$ for $C^{0}(I)$. For each $g \in C[0,1]$, set $\|g\|=\max _{x \in[0,1]}|g(x)|$.

In several inequalities $C$ denotes a generic constant that depends on the data of the boundary value problem (1.5) and possibly on the mesh grading but is independent of the mesh diameter when (1.5) is solved numerically; note that $C$ can take different values in different places.

1.1. Basic definitions. For $n=1,2, \ldots$ we denote by $A^{n}[0,1]$ the set of functions $g \in C^{n-1}[0,1]$ with $g^{(n-1)}$ absolutely continuous on $[0,1]$, i.e., $g^{(n)}$ exists almost everywhere in $[0,1]$ and

$$
g^{(n-1)}(x)=g^{(n-1)}(0)+\int_{t=0}^{x} g^{(n)}(t) d t \quad \text { for } 0 \leq x \leq 1 .
$$

Clearly $C^{n}[0,1] \subset A^{n}[0,1]$.

Let $\delta \in(0,1)$. For all $g \in L_{1}[0,1]$, as in [3] define the Riemann-Liouville fractional integral operator of order $\delta$ by

$$
\left(J^{\delta} g\right)(x)=\left[\frac{1}{\Gamma(\delta)} \int_{t=0}^{x}(x-t)^{\delta-1} g(t) d t\right] \quad \text { for } \quad 0 \leq x \leq 1 .
$$

Let $m$ be a positive integer. For any suitable function $g$, the Riemann-Liouville fractional derivative $D^{m-\delta}$ is defined [3, Definition 2.2] by

$$
D^{m-\delta} g(x)=\left(\frac{d}{d x}\right)^{m}\left(J^{\delta} g\right)(x) \text { for } 0<x \leq 1,
$$

and the Caputo fractional derivative $D_{*}^{m-\delta}$ is defined [3, Definition 3.2] in terms of $D^{m-\delta}$ by

$$
D_{*}^{m-\delta} g=D^{m-\delta}\left[g-T_{m-1}[g ; 0]\right],
$$

where $T_{m-1}[g ; 0]$ denotes the Taylor polynomial of degree $m-1$ of the function $g$ expanded around $x=0$. If $g \in A^{m}[0,1]$, then both these derivatives are well defined and by [3, Theorem 3.1] one also has the equivalent formulation

$$
D_{*}^{m-\delta} g(x):=\frac{1}{\Gamma(\delta)} \int_{t=0}^{x}(x-t)^{\delta-1} g^{(m)}(t) d t \quad \text { for } 0<x \leq 1 .
$$

Note that if one sets $\delta=0$ and $m=1$, then the Riemann-Liouville derivative $D^{1} \equiv D$ becomes the classical differential operator $d / d x$.

1.2. The boundary value problem. In this paper we consider the RiemannLiouville two-point boundary value problem

$$
\begin{aligned}
L u:=-D^{2-\delta} u+(b u)^{\prime}+c u & =f \quad \text { on }(0,1) \\
u(0)=0, \quad \alpha u(1)+\beta u^{\prime}(1) & =\gamma,
\end{aligned}
$$


where $0<\delta<1$, the functions $b, c, f$ lie in $C^{q}[0,1]$ for some positive integer $q$, and $\beta, \gamma \in \mathbb{R}$ with $\alpha \geq 0, \beta \geq 0$ and $\alpha+\beta>0$.

The choice of the homogeneous Dirichlet boundary condition $u(0)=0$ in $(1.5 \mathrm{~b})$ is motivated by the following example.

EXAMPLE 1.1. Consider $-D^{2-\delta} u=1$ on $(0,1)$, with the boundary conditions (1.5b). The general solution of this differential equation is, by [3, Example 2.4],

$$
u(x)=-\frac{x^{2-\delta}}{\Gamma(1-\delta)}+c_{1} x^{1-\delta}+c_{2} x^{-\delta}
$$

for some constants $c_{1}$ and $c_{2}$. The boundary condition $u(0)=0$ forces $c_{2}=0$. This is desirable as $c_{2} \neq 0$ would imply $u \notin C[0,1]$, thereby making the problem much more difficult to analyse. Then the boundary condition at $x=1$ will determine the value of $c_{1}$. In general one has $c_{1} \neq 0$, so $u \in C[0,1] \cap C^{1}(0,1]$ but $u \notin C^{1}[0,1]$. In fact $u^{\prime}(x)$ blows up at $x=0$. One has $u \in A^{1}[0,1]$ but $u \notin A^{2}[0,1]$.

In $(1.5)$ the convection term $(b u)^{\prime}$ is written in conservative form for our later convenience. The nonconservative form $b u^{\prime}$ can be rewritten as $(b u)^{\prime}-b^{\prime} u$ to fit into this framework.

Notation. Throughout the paper $C$ is used as a generic constant that depends on the data of the problem (1.5) but is independent of $x \in[0,1]$ and of the mesh diameter in the numerical method. The Lebesgue space $L_{1}[0,1]$ is used occasionally.

2. Reformulation of the boundary value problem. Our aim here is to reformulate (1.5) in terms of Volterra integral equations in order to show existence, uniqueness and regularity of a solution to (1.5), and furthermore to facilitate its efficient numerical solution. A related reformulation was used in [6], where a Caputo boundary value problem was rewritten in terms of the continuous variable $u^{\prime}$, but in (1.5) - as we saw in Example 1.1 - one may have $u^{\prime} \notin C[0,1]$, which would not fit with the standard Volterra theory in [1] so a different reformulation will be necessary here. Thus we shall rewrite (1.5) in terms of a lower-order fractional derivative of $u$ that lies in $C[0,1]$. Fundamentally, the Riemann-Liouville boundary value problem (1.5) is less well behaved than the analogous Caputo problem of [6] and requires more work for its satisfactory analysis and accurate numerical solution.

2.1. Derivation of integral equations from (1.5). We begin with the following helpful observation.

Lemma 2.1. Let $r \in A^{1}[0,1]$ with $r(0)=0$. Assume that $D^{1-\delta} r(x)$ is absolutely continuous on $[0,1]$. Then $D^{2-\delta} r$ exists almost everywhere in $[0,1]$ and $D^{2-\delta} r=$ $D D_{*}^{1-\delta} r$.

Proof. Lemma 2.12 and Theorem 3.1 of [3] tell us that $D^{1-\delta} r$ and $D_{*}^{1-\delta} r$ exist almost everywhere in $[0,1]$. By [3, Lemma 3.5] and the hypothesis $r(0)=0$, one has $D^{1-\delta} r=D_{*}^{1-\delta} r$. The derivative of an absolutely continuous function exists almost everywhere, so the definition (1.2) yields $D^{2-\delta} r=D D^{1-\delta} r=D D_{*}^{1-\delta} r$ almost everywhere in $[0,1]$.

Returning to (1.5), assume that $u \in A^{1}[0,1]$ and set

$$
\hat{u}=D_{*}^{1-\delta} u \text {. }
$$

Then $\hat{u}$ exists almost everywhere in $[0,1]$ by $[3$, Theorem 3.1$]$. Note that

$$
J^{1-\delta} \hat{u}(x)=u(x)-u(0)=u(x) \quad \text { for all } x \in(0,1]
$$


by $[3$, Theorem 3.8$]$ and (1.5b).

We shall use similar "hat" and "no hat" notation throughout the paper whenever a pair of functions are related by $D_{*}^{1-\delta}$ or $D^{1-\delta}$ or $J^{1-\delta}$.

Define the functions $\hat{z}$ and $\hat{v}$ by the weakly singular Volterra integral equations

$$
\begin{aligned}
& \hat{z}(x)-b(x) J^{1-\delta} \hat{z}(x)-\int_{t=0}^{x} c(t) J^{1-\delta} \hat{z}(t) d t=1 \quad \text { on }[0,1], \\
& \hat{v}(x)-b(x) J^{1-\delta} \hat{v}(x)-\int_{t=0}^{x} c(t) J^{1-\delta} \hat{v}(t) d t=F(x) \quad \text { on }[0,1],
\end{aligned}
$$

where $F(x):=\int_{0}^{x} f$.

Let $C^{q, \delta}(0,1]$ denote the space of functions $y \in C[0,1] \cap C^{q}(0,1]$ such that $|y(x)| \leq$ $C$ and $\left|y^{(k)}(x)\right| \leq C x^{(1-\delta)-k}$ for $k=1, \ldots, q$. By an argument similar to the proof of [6, Lemma 2.1], the functions $\hat{z}$ and $\hat{v}$ are well defined and lie in $C^{q, \delta}(0,1]$.

Furthermore, (2.3) and (2.4) imply that

$$
\hat{z}(0)=1 \quad \text { and } \quad \hat{v}(0)=0 .
$$

TheOREm 2.2. Let $u$ be a solution of (1.5). Assume that $u \in A^{1}[0,1]$ and $D^{1-\delta} u$ is absolutely continuous on $[0,1]$. Then for all $x \in[0,1]$ one has $u(x)=J^{1-\delta} \hat{u}(x)$ and

$$
\hat{u}(x)=\hat{u}(0) \hat{z}(x)-\hat{v}(x)
$$

Proof. By (2.2) we have $u(x)=J^{1-\delta} \hat{u}(x)$. Lemma 2.1 shows that $D^{2-\delta} u=D \hat{u}$ almost everywhere and $\hat{u}=D_{*}^{1-\delta} u=D^{1-\delta} u$ is absolutely continuous on $[0,1]$. Thus one can integrate (1.5a) from 0 to $x \in(0,1]$, obtaining

$$
\hat{u}(x)-(b u)(x)-\int_{t=0}^{x} c(t) u(t) d t=\hat{u}(0)-F(x) .
$$

From (2.2) and (2.7) we get

$$
\hat{u}(x)-b(x) J^{1-\delta} \hat{u}(x)-\int_{t=0}^{x} c(t) J^{1-\delta} \hat{u}(t) d t=\hat{u}(0)-F(x) .
$$

The identity (2.6) now follows from the definitions of $\hat{z}$ and $\hat{v}$.

Equations (2.2) and (2.6) show that a solution $u$ of (1.5) that satisfies the hypotheses of Theorem 2.2 can be written as a linear combination of the solutions $\hat{z}$ and $\hat{v}$ of the Volterra integral equations (2.3) and (2.4).

The hypotheses of Theorem 2.2 are reasonable (cf. Example 1.1) and unrestrictive: they say only that $u^{\prime} \in L_{1}[0,1], D^{2-\delta} u \in L_{1}[0,1], u(x)=\int_{0}^{x} u^{\prime}$ and $D^{1-\delta} u(x)-$ $D^{1-\delta} u(0)=\int_{0}^{x} D^{2-\delta} u$.

2.2. Derivation of (1.5) from integral equations. As a converse to Theorem 2.2 we show now that, starting from the definitions of $\hat{z}$ and $\hat{v}$, one can choose $\hat{u}(0)$ in such a way that (2.6) and (2.2) yield a solution of (1.5).

At various points in this section, we shall assume one or both of the inequalities

$$
\begin{aligned}
c \geq 0 & \text { on }[0,1], \\
b^{\prime}+c \geq 0 & \text { on }[0,1] .
\end{aligned}
$$


Conditions such as these are commonly assumed in classical second-order differential equations to ensure that the differential operator $L$ and its formal adjoint satisfy a maximum principle.

In particular, to satisfy the boundary condition (1.5b) at $x=1$, by $(2.2)$ and (2.6) one needs

$$
\begin{aligned}
\gamma & =\alpha u(1)+\beta u^{\prime}(1) \\
& =\alpha J^{1-\delta} \hat{u}(1)+\beta\left[D J^{1-\delta} \hat{u}(1)\right] \\
& =\alpha\left[\hat{u}(0) J^{1-\delta} \hat{z}(1)-J^{1-\delta} \hat{v}(1)\right]+\beta\left[\hat{u}(0) D J^{1-\delta} \hat{z}(1)-D J^{1-\delta} \hat{v}(1)\right] .
\end{aligned}
$$

Here $\alpha, \beta, \gamma$ are known with $\alpha \geq 0, \beta \geq 0$ and $\alpha+\beta>0$. One can solve (2.10) for $\hat{u}(0)$ if

$$
\alpha J^{1-\delta} \hat{z}(1)+\beta D J^{1-\delta} \hat{z}(1)>0 .
$$

No condition is required here for the function $\hat{v}$.

We shall prove that $(2.11)$ is true. Set $z(x)=J^{1-\delta} \hat{z}(x)$ for $x \in[0,1]$. Clearly $z(0)=0$. We know that $\hat{z} \in C[0,1] \subset L_{1}[0,1]$, so by $[3$, Theorem 2.14] one has

$$
D^{1-\delta} z=\hat{z} \text {. }
$$

EXAMPLE 2.1. If $b=\lambda \in \mathbb{R}$ is constant and $c \equiv 0$, then a calculation yields

$$
\hat{z}(x)=E_{1-\delta, 1}\left(\lambda x^{1-\delta}\right), z(x)=x^{1-\delta} E_{1-\delta, 2-\delta}\left(\lambda x^{1-\delta}\right), z^{\prime}(x)=x^{-\delta} E_{1-\delta, 1-\delta}\left(\lambda x^{1-\delta}\right),
$$

where the two-parameter Mittag-Leffler function $E$ is defined by

$$
E_{\mu, \theta}(z)=\sum_{k=0}^{\infty} \frac{z^{k}}{\Gamma(\mu k+\theta)} \quad \text { for } \mu, \theta, z \in \mathbb{R} \text { with } \mu>0 .
$$

See [3, 7] for discussions of this function. These formulas, combined with known properties of the Mittag-Leffler function, imply that for any $\lambda \in \mathbb{R}$ one has $z(x)>0$ and $z^{\prime}(x)>0$ for $x>0$, and $z^{\prime}\left(0^{+}\right)=+\infty$.

Lemma 2.3. Assume condition (2.9a). Then one has $z(x)>0$ for all $x \in(0,1]$.

Proof. As $D^{1-\delta} z=\hat{z}$, equation (2.3) implies that

$$
D^{1-\delta} z(x)-(b z)(x)-\int_{t=0}^{x} c(t) z(t) d t=1 \text { on }(0,1] .
$$

Suppose that the conclusion of the lemma is false. Set $x^{*}=\inf \{x \in(0,1]: z(x) \leq$ $0\}$. Now $\hat{z}(0)=1$ and $\hat{z} \in C[0,1]$ imply that $z(x)=J^{1-\delta} \hat{z}(x)>0$ for all positive $x$ sufficiently close to 0 , so $0<x^{*} \leq 1$. Furthermore, $z\left(x^{*}\right)=0$. Thus at $x=x^{*}$, the equation (2.14) becomes

$$
\begin{aligned}
1+\int_{t=0}^{x^{*}} c(t) z(t) d t=D^{1-\delta} z\left(x^{*}\right) & =\left.\frac{d}{d x}\left(\frac{1}{\Gamma(\delta)} \int_{t=0}^{x}(x-t)^{\delta-1} z(t) d t\right)\right|_{x=x^{*}} \\
& =\frac{\delta-1}{\Gamma(\delta)} \int_{t=0}^{x^{*}}\left(x^{*}-t\right)^{\delta-2} z(t) d t
\end{aligned}
$$

as can be seen by integrating by parts before and after applying $d / d x$, and using the property $|z(t)| \leq C\left(x^{*}-t\right)$ for $0 \leq t \leq x^{*}$ which follows from $z\left(x^{*}\right)=0$ and 
$z \in C[0,1] \cap C^{q}(0,1]$. But $\delta-1<0$ and $z(t)>0$ for $0<t<x^{*}$, so the right-hand side of the equation is negative while the left-hand side is positive by (2.9a). From this contradiction we infer that the lemma is true.

Lemma 2.4. Assume condition (2.9). Then one has $z \in C^{1}(0,1]$ and $z^{\prime}(x)>0$ for all $x \in(0,1]$.

Proof. By a change of variable in the definition of $z$ one gets

$$
z(x)=\frac{1}{\Gamma(1-\delta)} \int_{t=0}^{x} t^{-\delta} \hat{z}(x-t) d t
$$

Differentiating and using $\hat{z}(0)=1$,

$$
s(x):=z^{\prime}(x)=\frac{1}{\Gamma(1-\delta)}\left[x^{-\delta}+\int_{t=0}^{x} t^{-\delta} \hat{z}^{\prime}(x-t) d t\right] \quad \text { for } 0<x \leq 1 .
$$

Clearly $z \in C^{1}(0,1]$. Note that $\hat{z} \in C^{q, \delta}(0,1]$ implies that $\left|\hat{z}^{\prime}(t)\right| \leq C t^{-\delta}$ for some constant $C$. Now a standard estimate [3, Theorem D.6] for the Euler Beta function yields

$$
\left|x^{\delta} s(x)-\frac{1}{\Gamma(1-\delta)}\right| \leq C x^{\delta} \int_{t=0}^{x} t^{-\delta}(x-t)^{-\delta} d t \leq C x^{1-\delta}
$$

and it follows that $s(x)>0$ for all positive $x$ sufficiently close to zero.

Since $z(0)=0$, we have $D^{1-\delta} z=D_{*}^{1-\delta} z$ by [3, Lemma 3.4]. Consequently $D D^{1-\delta} z=D D_{*}^{1-\delta} z=D^{1-\delta} s$ by the definitions (1.2) and (1.3). Thus differentiating (2.14) yields

$$
D^{1-\delta} s(x)=\left[b s+\left(b^{\prime}+c\right) z\right](x) \quad \text { on }(0,1] .
$$

Suppose that the conclusion of the lemma is false. Set $x^{*}=\inf \{x \in(0,1]: s(x) \leq 0\}$. Then $x^{*} \in(0,1]$ since $s(x)>0$ near $x=0$. We now derive a contradiction in $(2.15)$ at $x=x^{*}$ by imitating the proof of Lemma 2.3, but with the argument modified to handle the complication that $s(x)$ blows up as $x \rightarrow 0$. Choose $\bar{x} \in\left(0, x^{*}\right)$. Then

$$
\begin{aligned}
\left.D^{1-\delta} s(x)\right|_{x=x^{*}}=\frac{d}{d x}( & \left.\frac{1}{\Gamma(\delta)} \int_{t=0}^{\bar{x}}(x-t)^{\delta-1} s(t) d t\right)\left.\right|_{x=x^{*}} \\
& \quad+\left.\frac{d}{d x}\left(\frac{1}{\Gamma(\delta)} \int_{t=\bar{x}}^{x}(x-t)^{\delta-1} s(t) d t\right)\right|_{x=x^{*}} \\
= & \frac{\delta-1}{\Gamma(\delta)}\left[\int_{t=0}^{\bar{x}}\left(x^{*}-t\right)^{\delta-2} s(t) d t+\int_{t=\bar{x}}^{x^{*}}\left(x^{*}-t\right)^{\delta-2} s(t) d t\right] ;
\end{aligned}
$$

here the differentiation of the first integral is routine while for the second we integrate by parts, then differentiate, then integrate by parts again. As $s(x)>0$ on $\left(0, x^{*}\right)$ we deduce that $\left.D^{1-\delta} s(x)\right|_{x=x^{*}}<0$. But this contradicts (2.15) at $x=x^{*}$ since $s\left(x^{*}\right)=0$ by the definition of $x^{*}$ and $\left[\left(b^{\prime}+c\right) z\right]\left(x^{*}\right)>0$ by Lemma 2.3 and $(2.9 \mathrm{~b})$. This concludes the proof.

Lemmas 2.3 and 2.4 imply that the condition (2.11), which can be written as $\alpha z(1)+\beta z^{\prime}(1)>0$, is satisfied.

THEOREM 2.5. [Existence of a solution to the Riemann-Liouville boundary value problem] Assume both conditions in (2.9). Let $\hat{z}$ and $\hat{v}$ be defined by (2.3) and (2.4). 
Define $\hat{u}(0)$ by

$$
\hat{u}(0):=\frac{\gamma+\alpha J^{1-\delta} \hat{v}(1)+\beta D J^{1-\delta} \hat{v}(1)}{\alpha J^{1-\delta} \hat{z}(1)+\beta D J^{1-\delta} \hat{z}(1)} .
$$

For $x \in[0,1]$, define $\hat{u}(x)$ by $(2.6)$ and $u(x)$ by $(2.2)$. Then

$$
u(x)=J^{1-\delta} \hat{u}(x)=\hat{u}(0) J^{1-\delta} \hat{z}(x)-J^{1-\delta} \hat{v}(x)
$$

is a solution of (1.5).

Proof. Recall that $\alpha \geq 0, \beta \geq 0$ and $\alpha+\beta>0$. From Lemmas 2.3 and 2.4 it follows that the denominator of $(2.16)$ is positive so $\hat{u}(0)$ is well defined. It is now clear that $\hat{u}(x)$ and $u(x)$ are well defined.

By construction $\hat{u} \in C[0,1]$ so $(2.2)$ implies that $u(0)=0$. The definition of $\hat{u}(0)$ and the calculation leading to $(2.10)$ yield $\alpha u(1)+\beta u^{\prime}(1)=\gamma$. Thus it remains only to show that $u$ satisfies $(1.5 \mathrm{a})$. For $0<x \leq 1$,

$$
\begin{aligned}
D^{2-\delta} u(x) & =\left(D D^{1-\delta}\right) J^{1-\delta} \hat{u}(x)=D \hat{u}(x) \\
& =\hat{u}(0) \hat{z}^{\prime}(x)-\hat{v}^{\prime}(x) \\
& =\frac{d}{d x}\left[b(x) J^{1-\delta} \hat{u}(x)\right]+c(x) J^{1-\delta} \hat{u}(x)-f(x) \\
& =(b u)^{\prime}(x)+c(x) u(x)-f(x),
\end{aligned}
$$

where we used [3, Theorem 2.14] and the definitions of $\hat{u}, \hat{z}, \hat{v}$ and $u$. That is, $u$ satisfies (1.5a) and we are done.

REMARK 2.1. In the case where (1.5) has a Dirichlet boundary condition at $x=1$ (i.e., when $\beta=0$ ), Lemma 2.4 is no longer needed and consequently the hypothesis $(2.9 \mathrm{~b})$ can be removed from Theorem 2.5. Similarly, one does not need the condition $(2.9 \mathrm{~b})$ in the remainder of the paper when $\beta=0$.

Corollary 2.6. [pointwise bounds on derivatives of $u$ ] There exists a constant $C$ such that $\|u\| \infty \leq C$ and

$$
\left|u^{(i)}(x)\right| \leq C x^{(1-\delta)-i} \quad \text { for } i=1,2, \ldots, q \text { and } \quad x \in(0,1] .
$$

Proof. Recall that the functions $\hat{z}, \hat{v}$ lie in $C^{q, \delta}(0,1]$. Invoking [2, Lemma 2.2], it follows that $J^{1-\delta} \hat{z}$ and $J^{1-\delta} \hat{v} \in C^{q, \delta}(0,1]$. Thus for some constant $C$ we have

$$
\left|J^{1-\delta} \eta(x)\right| \leq C \text { and }\left|\left(J^{1-\delta} \eta\right)^{(i)}(x)\right| \leq C x^{(1-\delta)-i} \text { for } i=1,2, \ldots, q \text { and } x \in(0,1]
$$

where $\eta$ can be $\hat{z}$ or $\hat{v}$. The desired bound now follows from (2.16) and (2.17).

Example 1.1 shows that the bounds of Corollary 2.6 are sharp. Nevertheless this corollary does not give us a complete picture of the regularity of $u$, because $D^{2-\delta} g$ is not defined for all functions $g$ that satisfy (2.19) yet $D^{2-\delta} u$ is defined (it appears in (1.5a)). Remark 2.2 will shed more light on the properties of $u$, but first we derive a technical result that will prove useful in discussing $u$ here and in constructing a numerical method for solving (1.5) in $\S 3.1$.

LEMma 2.7. Let the function $\phi$ satisfy

$$
\phi(x)-b(x) J^{1-\delta} \phi(x)-\int_{t=0}^{x} c(t) J^{1-\delta} \phi(t) d t=\psi(x) \quad \text { on }[0,1],
$$


where $\psi^{\prime} \in C^{q, \delta}(0,1]$ and $\psi(0)=0$. Then the functions $\phi, J^{1-\delta} \phi, \phi^{\prime}, J^{1-\delta}\left(\phi^{\prime}\right)$ all lie in $C^{q, \delta}(0,1]$,

$$
D J^{1-\delta} \phi(x)=J^{1-\delta} \phi^{\prime}(x) \quad \text { for } 0<x \leq 1,
$$

and

$$
\phi^{\prime}(x)-\left(b J^{1-\delta} \phi^{\prime}\right)(x)=\psi^{\prime}(x)+\left(\left(b^{\prime}+c\right) J^{1-\delta} \phi\right)(x) \quad \text { on }[0,1]
$$

Proof. First, $\psi^{\prime} \in C^{q, \delta}(0,1]$ trivially implies that $\psi \in C^{q, \delta}(0,1]$. Then using (2.20), an argument similar to the proof of [6, Lemma 2.1] shows that (2.20) has a unique solution $\phi \in C^{q, \delta}(0,1]$. Hence $J^{1-\delta} \phi \in C^{q, \delta}(0,1]$ by [2, Lemma 2.2].

Now $(2.20)$ and $\psi(0)=0$ imply that $\phi(0)=0$. As $\left|\phi^{\prime}(x)\right| \leq C x^{-\delta}$, one can integrate by parts to get

$$
\begin{aligned}
J^{1-\delta} \phi(x) & =\frac{1}{\Gamma(1-\delta)} \int_{t=0}^{x}(x-t)^{-\delta} \phi(t) d t=\frac{1}{\Gamma(2-\delta)} \int_{t=0}^{x}(x-t)^{1-\delta} \phi^{\prime}(t) d t \\
& =J^{2-\delta} \phi^{\prime}(x) .
\end{aligned}
$$

This equation can be differentiated immediately to give (2.21). Then differentiating $(2.20)$ yields $(2.22)$ on $(0,1]$. The proof of [6, Lemma 2.1], applied to $(2.22)$, shows that $\phi^{\prime} \in C^{q, \delta}(0,1]$. Hence $(2.22)$ is valid on all of $[0,1]$, and another appeal to $[2$, Lemma 2.2] yields $J^{1-\delta} \phi^{\prime} \in C^{q, \delta}(0,1]$.

REMARK 2.2. [Fine regularity of $u$ ] Example 1.1 shows that $u$ may not lie in $A^{2}[0,1]$, but the differential equation (1.5a) that $u$ satisfies includes the term $D^{2-\delta} u$ which is frequently perceived to be well defined only when $u \in A^{2}[0,1]$. This anomaly arises because $u$ may have a singular component that lies outside $A^{2}[0,1]$ but is annihilated by the differential operator-for instance, the $x^{1-\delta}$ term in Example 1.1.

The decomposition (2.2) and (2.6), stating that

$$
u(x)=J^{1-\delta} \hat{u}(x)=\hat{u}(0) J^{1-\delta} \hat{z}(x)-J^{1-\delta} \hat{v}(x),
$$

illustrates this structure. For we show now that $J^{1-\delta} \hat{v}$ lies in $A^{2}[0,1]$ but $z$ is less smooth-it is not in $C^{1}[0,1]$ and therefore not in $A^{2}[0,1]$ - yet $z$ satisfies the homogeneous analogue of $(1.5 \mathrm{a})$.

From equation (2.4) one sees that $\hat{v}$ satisfies the hypotheses of Lemma 2.7. Thus $J^{1-\delta} \hat{v}^{\prime} \in C^{q, \delta}(0,1]$ and it now follows from $(2.21)$ that $J^{1-\delta} \hat{v} \in A^{2}[0,1]$.

On the other hand, a calculation similar to the proof of Lemma 2.7 applied to $\hat{z}$ will give, instead of (2.23), the formula

$$
J^{1-\delta} \hat{z}(x)=\frac{x^{1-\delta}}{\Gamma(2-\delta)}+J^{2-\delta} \hat{z}^{\prime}(x)
$$

because $\hat{z}(0)=1$. Hence $\left(J^{1-\delta} \hat{z}\right)^{\prime}(x)$ blows up as $x \rightarrow 0$, which implies that $J^{1-\delta} \hat{z}$ cannot lie in $A^{2}[0,1]$. But the calculation yielding (2.18), when one begins from $J^{1-\delta} \hat{z}$ instead of $J^{1-\delta} \hat{u}$, demonstrates that

$$
D^{2-\delta} J^{1-\delta} \hat{z}=\left(b J^{1-\delta} \hat{z}\right)^{\prime}(x)+c(x) J^{1-\delta} \hat{z}(x),
$$

i.e., $J^{1-\delta} \hat{z}$ is annihilated by the differential operator of (1.5a).

This decomposition of $u$ will be explored further in Lemma 3.1. 
2.3. Uniqueness of solution to (1.5). The earlier results enable us to prove a uniqueness result for (1.5).

THEOREM 2.8. Let $u_{1}$ and $u_{2}$ be solutions of (1.5) with $u_{1}, u_{2} \in A^{1}[0,1]$ and $D^{1-\delta} u_{1}, D^{1-\delta} u_{2}$ absolutely continuous on $[0,1]$. Then $u_{1} \equiv u_{2}$.

Proof. Imitating (2.1), set $\hat{u}_{1}=D_{*}^{1-\delta} u_{1}$ and $\hat{u}_{2}=D_{*}^{1-\delta} u_{2}$. Theorem 2.2 implies that

$$
u_{i}(x)=J^{1-\delta} \hat{u}_{i}(x) \text { and } \hat{u}_{i}(x)=\hat{u}_{i}(0) \hat{z}(x)-\hat{v}(x) \text { for } i=1,2 \text { and } x \in[0,1] .
$$

But the calculation (2.10) shows that one must choose $\hat{u}_{1}(0)=\hat{u}_{2}(0)=\hat{u}(0)$ in $(2.16)$ to enable $u_{1}$ and $u_{2}$ to satisfy the boundary condition at $x=1$ (Lemmas 2.3 and 2.4 imply that the formula $(2.16)$ is well defined). Now $(2.24)$ yields $\hat{u}_{1}(x)=\hat{u}_{2}(x)$ and $u_{1}(x)=u_{2}(x)$ for all $x \in[0,1]$.

REMARK 2.3. [Existence and uniqueness for a Caputo two-point boundary value problem] In [6] a result similar to the analysis of §2.1 and §2.2 was shown for a related two-point boundary value problem where the Riemann-Liouville derivative in (1.5a) is replaced by a Caputo derivative: it was shown that the solution of the original problem can be expressed as an integral of a linear combination of the solutions to two weakly singular Volterra integral equations. One can easily deduce from the proof of that equivalence an existence result for the Caputo problem that is analogous to Theorem 2.5 and a uniqueness result analogous to Theorem 2.8. Furthermore, both these results require (in the notation of [6]) only $\alpha_{0} \geq 0$ instead of the more restrictive hypothesis $\alpha_{0} \geq 1 /(1-\delta)$ that was used throughout that paper.

3. Two numerical methods for solving (1.5). In this section we present and analyse two numerical methods for solving (1.5) numerically for $u$ in an efficient way. Both methods are based on the representation (2.17) of Theorem 2.5, which is the following:

$$
u(x)=\hat{u}(0) J^{1-\delta} \hat{z}(x)-J^{1-\delta} \hat{v}(x) \quad \text { for } x \in[0,1] .
$$

Thus $\hat{u}(0), J^{1-\delta} \hat{z}(x)$ and $J^{1-\delta} \hat{v}(x)$ need to be computed numerically.

In both methods, the solution of (1.5) is reduced to solving an independent pair of weakly singular Voterra integral equations. This is done by employing the collocation method of [6], which uses piecewise polynomials of degree $m-1$; for completeness this method is described in detail in the Appendix below.

When (1.5) was presented, we assumed that the functions $b, c, f$ lay in $C^{q}[0,1]$ for some positive integer $q$. In $\S 3$ we assume that $q \geq m+1$ to permit us to appeal to the convergence results of [6, Corollaries 3.1 and 3.2].

In the error estimates of $\S 3$, the generic constants $C$ depend on the choice of collocation parameters $\left\{c_{j}\right\}$ and on the mesh grading $\rho$, but are independent of the mesh diameter $h$.

3.1. Method I: singularity subtraction. The method of this subsection subtracts from $u$ its principal singular component, thereby reducing the solution of (1.5) to the numerical solution of two less singular Volterra integral equations that fit into the framework of $[6]$.

Set

$$
v=J^{1-\delta} \hat{v} \quad \text { and } \quad V=v^{\prime} .
$$

In (3.1) we need to deal with $J^{1-\delta} \hat{z}$ as well as $J^{1-\delta} \hat{v}$, but Remark 2.2 reveals that $J^{1-\delta} \hat{z}$ is worse behaved than $J^{1-\delta} \hat{v}$ : while $\hat{z}$ satisfies $(2.3)$, one has $\hat{z}(0)=1 \neq 0$ so $\hat{z}$ 
fails to satisfy the hypotheses of Lemma 2.7 (unlike $\hat{v}$ ). To enable us to appeal to this lemma, we shall modify $\hat{z}$ by subtracting off the essential singularity that $J^{1-\delta} \hat{z}(x)$ has at $x=0$.

Set

$$
\lambda=b(0), \hat{z}_{0}(x)=E_{1-\delta, 1}\left(\lambda x^{1-\delta}\right), s=J^{1-\delta}\left(\hat{z}-\hat{z}_{0}\right) \text { and } S=s^{\prime},
$$

where the Mittag-Leffler function $E$ was defined in (2.13) and $0 \leq x \leq 1$.

For any suitable function $W$, set

$$
(\mathcal{I} W)(x)=W(x)-J^{1-\delta}(b W)(x)-J^{1-\delta}\left(\left(b^{\prime}+c\right) \int_{0}^{(\cdot)} W\right)(x) \quad \text { for } x \in[0,1] .
$$

LEMma 3.1. [Exact solution decomposition] Under condition (2.9), the unique solution $u$ of (1.5) can be decomposed according to equations (2.16), (2.17), (3.2) and (3.3), in which $v$ and $s$ are in $A^{2}[0,1]$ and the functions $V$ and $S$ defined in (3.2) and (3.3) satisfy the Volterra integral equations

$$
(\mathcal{I} V)(x)=J^{1-\delta} f(x)
$$

and

$$
(\mathcal{I} S)(x)=J^{1-\delta}\left\{\left[(b-\lambda) J^{1-\delta} \hat{z}_{0}\right]^{\prime}+c J^{1-\delta} \hat{z}_{0}\right\}(x)
$$

for $x \in[0,1]$.

Proof. It is shown in Remark 2.2 that $v=J^{1-\delta} \hat{v} \in A^{2}[0,1]$. The same argument yields $s \in A^{2}[0,1]$ by its construction.

Now consider (3.5). By its definition one has $v(0)=0$. Equation (2.4) shows that $\hat{v}$ satisfies the hypotheses of Lemma 2.7. Thus equations (2.21) and (2.22) yield

$$
\hat{v}^{\prime}(x)-\left(b v^{\prime}\right)(x)-\left(\left(b^{\prime}+c\right) v\right)(x)=f(x) \quad \text { on }[0,1] .
$$

Apply $J^{1-\delta}$ to this and again invoke (2.21) to get

$$
v^{\prime}-J^{1-\delta}\left(b v^{\prime}\right)-J^{1-\delta}\left(\left(b^{\prime}+c\right) v\right)=J^{1-\delta} f \quad \text { on }[0,1],
$$

which is (3.5).

Next, consider (3.6). It is straightforward to verify that $\hat{z}_{0} \in C^{q, \delta}(0,1]$. Now

$$
\hat{z}_{0}(x)-\lambda J^{1-\delta} \hat{z}_{0}(x)=1 \quad \text { for } 0 \leq x \leq 1
$$

by [1, Theorem 6.1.1]. Subtracting this equation from (2.3) gives

$$
\left(\hat{z}-\hat{z}_{0}\right)(x)-b J^{1-\delta}\left(\hat{z}-\hat{z}_{0}\right)(x)-\int_{0}^{x} c J^{1-\delta}\left(\hat{z}-\hat{z}_{0}\right)=(b-\lambda) J^{1-\delta}\left(\hat{z}_{0}\right)+\int_{0}^{x} c J^{1-\delta} \hat{z}_{0}
$$

for $x \in[0,1]$. Now $\hat{z}-\hat{z}_{0} \in C^{q, \delta}(0,1]$ and $\left(\hat{z}-\hat{z}_{0}\right)(0)=1-1=0$ so by $(3.7)$ we can apply Lemma 2.7 with $\phi=\hat{z}-\hat{z}_{0}$. Equations $(2.21)$ and $(2.22)$ yield $s^{\prime}=J^{1-\delta}\left(\hat{z}-\hat{z}_{0}\right)^{\prime}$ and

$$
\left(\hat{z}-\hat{z}_{0}\right)^{\prime}-b s^{\prime}-\left(b^{\prime}+c\right) s=\left[(b-\lambda) J^{1-\delta} \hat{z}_{0}\right]^{\prime}+c J^{1-\delta} \hat{z}_{0} \quad \text { on }[0,1] .
$$

Applying $J^{1-\delta}$ to this equation yields (3.6). 
Recalling that $\lambda=b(0)$ and $J^{1-\delta}$ maps $C^{q, \delta}(0,1]$ to itself by [2, Lemma 2.2], one can see easily that $J^{1-\delta} f$ and $\left[(b-\lambda) J^{1-\delta} \hat{z}_{0}\right]^{\prime}+c J^{1-\delta} \hat{z}_{0}$ lie in $C^{q, \delta}(0,1]$. Thus (3.5) and (3.6) have exactly the same form and regularity as the integral equation that was studied in [6] (see equation (2.4) there) and solved numerically using the iterated collocation method on a graded mesh of diameter $h$ with piecewise polynomials of degree $m-1 \geq 0$ lying in the space $S_{m-1}^{-1}$ defined in (A.2), where the value of $m$ is chosen by the user; a full description of this method is given in the Appendix below.

Set

$$
v_{h}(x)=\int_{0}^{x} V_{h}^{i t}(t) d t \quad \text { and } \quad s_{h}(x)=\int_{0}^{x} S_{h}^{i t}(t) d t \quad \text { for } x \in[0,1] .
$$

Lemma 3.2. There exists a constant $C$ such that

$$
\left\|v-v_{h}\right\|+\left\|V-V_{h}^{i t}\right\|+\left\|s-s_{h}\right\|+\left\|S-S_{h}^{i t}\right\| \leq C\left(K h^{m}+h^{m+1-\delta}\right),
$$

where the quantity $K$ is defined in (A.7).

Proof. The desired bounds for $V_{h}^{i t}$ and $S_{h}^{i t}$ are immediate from (A.8). The bound on $\left\|v-v_{h}\right\|+\left\|s-s_{h}\right\|$ then follows from (3.2) and (3.8).

Finally, we can compute an accurate approximation of $u$.

Theorem 3.3. Assume both conditions in (2.9). Set $Z_{h}=S_{h}^{i t}+\left(J^{1-\delta} \hat{z}_{0}\right)^{\prime}$ and $z_{h}(x)=1+\int_{0}^{x} Z_{h}=s_{h}(x)+\left(J^{1-\delta} \hat{z}_{0}\right)(x)$ for $x \in[0,1]$. Set

$$
\hat{u}_{h}(0)=\frac{\gamma+\alpha v_{h}(1)+\beta V_{h}^{i t}(1)}{\alpha z_{h}(1)+\beta Z_{h}(1)}
$$

and

$$
u_{h}(x)=\hat{u}_{h}(0) z_{h}(x)-v_{h}(x) \quad \text { for } x \in(0,1] .
$$

Then for $h$ sufficiently small, $\hat{u}_{h}(0)$ is well defined by (3.10) and

$$
\left\|u-u_{h}\right\| \leq C\left(K h^{m}+h^{m+1-\delta}\right)
$$

for some constant $C$.

Proof. We know $\left(J^{1-\delta} \hat{z}_{0}\right)(x)$ and $\left(J^{1-\delta} \hat{z}_{0}\right)^{\prime}(x)$ explicitly from (2.13) and (3.3). Thus, appealing to Lemma 3.2 , one has

$$
\left\|D J^{1-\delta} \hat{z}-Z_{h}\right\|+\left\|J^{1-\delta} \hat{z}-z_{h}\right\| \leq C\left(K h^{m}+h^{m+1-\delta}\right) .
$$

Hence Lemmas 2.3, 2.4 and 3.2 ensure that the denominator of (3.10) is nonzero for $h$ sufficiently small, and thus $\hat{u}_{h}(0)$ is well defined. Theorem 2.5, Lemma 3.2 and (3.13) imply that $\left|\hat{u}(0)-\hat{u}_{h}(0)\right| \leq C\left(K h^{m}+h^{m+1-\delta}\right)$ and furthermore that (3.12) holds true.

3.2. Method II: direct solution of (2.3) and (2.4). In this section we discuss an alternative numerical method for solving (3.1). It is based on computing approximations of $z=J^{1-\delta} \hat{z}$ and $v=J^{1-\delta} \hat{v}$ directly from (2.3) and (2.4)-i.e., unlike $\S 3.1$, approximations of $z$ and $v$ are not constructed from approximations of $z^{\prime}$ and $v^{\prime}$.

To begin, (2.3) and (2.4) are rewritten in a form that fits the analysis of [6].

LEMma 3.4. Consider the weakly singular Volterra integral equation

$$
\hat{\sigma}(x)-b(x) J^{1-\delta} \hat{\sigma}(x)-\int_{t=0}^{x} c(t) J^{1-\delta} \hat{\sigma}(t) d t=\zeta(x) \quad \text { on }[0,1],
$$


where $\hat{\sigma}$ is unknown, $\zeta$ is given and $b, c$ are as in (1.5a). Set $\sigma=J^{1-\delta} \hat{\sigma}$. Then $\sigma$ satisfies the weakly singular Volterra integral equation

$$
\sigma(x)-J^{1-\delta}\left(b \sigma-\int_{0}^{(\cdot)} c \sigma\right)(x)=J^{1-\delta} \zeta(x) \quad \text { on }[0,1] .
$$

Proof. The definition of $\sigma$ implies that (3.14) can be written as

$$
\hat{\sigma}(x)-b(x) \sigma(x)-\int_{t=0}^{x} c(t) \sigma(t) d t=\zeta(x)
$$

Applying $J^{1-\delta}$ to this equation, we get (3.15).

Equation (3.15) is almost identical to $[6,(2.4)]$ : the only difference is that in the latter one has $c \int_{0}^{(\cdot)} \sigma$ instead of $\int_{0}^{(\cdot)} c \sigma$. This minor change in the lowest-order term does not affect the analysis of [6].

Observe that equation (3.14) includes (2.3) and (2.4) as special cases. Thus, by virtue of Lemma 3.4, from (2.3) and (2.4) one gets

$$
z(x)-J^{1-\delta}\left(b z-\int_{0}^{(\cdot)} c z\right)(x)=\frac{x^{1-\delta}}{\Gamma(2-\delta)} \text { on }[0,1]
$$

and

$$
v(x)-J^{1-\delta}\left(b v-\int_{0}^{(\cdot)} c v\right)(x)=J^{1-\delta} F(x) \text { on }[0,1],
$$

respectively.

We are now in a position to compute a fresh approximation of $u$.

TheOrem 3.5. Assume both conditions in (2.9). Let $\tilde{z}_{h}$ and $\tilde{v}_{h}$ be piecewise polynomial approximations of degree $m-1$ of $z$ and $v$ that are obtained by applying the iterated collocation method described in the Appendix to (3.16) and (3.17) respectively. Apply backward differentiation of $\tilde{z}_{h}$ and $\tilde{v}_{h}$ at $x=1$ using $p \geq m+1$ nodal points $x_{i}$ (see Appendix for their definition) to generate approximations of $z^{\prime}(1)$ and $v^{\prime}(1)$ that we write as $\left(\nabla_{p, h} \tilde{z}_{h}\right)(1)$ and $\left(\nabla_{p, h} \tilde{v}_{h}\right)^{\prime}(1)$.

Assume that $m-1 \geq \min \{1, K \beta\}$ and set

$$
\tilde{u}_{h}(0)=\frac{\gamma+\alpha \tilde{v}_{h}(1)+\beta\left(\nabla_{p, h} \tilde{v}_{h}\right)(1)}{\alpha \tilde{z}_{h}(1)+\beta\left(\nabla_{p, h} \tilde{z}_{h}\right)(1)}
$$

and

$$
\tilde{u}_{h}(x)=\tilde{u}_{h}(0) \tilde{z}_{h}(x)-\tilde{v}_{h}(x) \quad \text { for } x \in(0,1] .
$$

Then for $h$ sufficiently small, $\tilde{u}_{h}(0)$ is well defined by (3.18) and

$$
\left\|u-\tilde{u}_{h}\right\| \leq C\left[\beta\left(K h^{m-1}+h^{m-\delta}\right)+K h^{m}+h^{m+1-\delta}\right]
$$

for some constant $C$, where the quantity $K$ is defined in (A.7).

Proof. By (A.5) and (A.8) one has

$$
\left\|z-\tilde{z}_{h}\right\|+\left\|v-\tilde{v}_{h}\right\| \leq C\left(K h^{m}+h^{m+1-\delta}\right) .
$$


The coarseness and smoothness of the mesh near $x=1$ then implies that

$$
\left|z^{\prime}(1)-\left(\nabla_{p, h} \tilde{z}_{h}\right)^{\prime}(1)\right|+\left|v^{\prime}(1)-\left(\nabla_{p, h} \tilde{v}_{h}\right)(1)\right| \leq C\left(K h^{m-1}+h^{m-\delta}\right) .
$$

Consequently $\alpha \tilde{z}_{h}(1)+\beta\left(\nabla_{p, h} \tilde{z}_{h}\right)^{\prime}(1) \neq 0$ for $h$ sufficiently small by Lemmas 2.3 and 2.4. It follows that the approximation (3.18) of (2.16) satisfies

$$
\left|u(0)-\tilde{u}_{h}(0)\right| \leq C\left[\beta\left(K h^{m-1}+h^{m-\delta}\right)+K h^{m}+h^{m+1-\delta}\right]
$$

and furthermore the approximation $\tilde{u}_{h}(x)$ satisfies (3.20).

Remark 3.1. [Method I versus Method II] The error bound for Method II that is proved in Theorem 3.5 is inferior to the error bound in Theorem 3.3 for Method I except when one has a Dirichlet boundary condition at $x=1$ in (1.5); on the other hand, Method II is simpler to implement. Thus when one has a Dirichlet condition at $x=1$, Method II is to be preferred. Furthermore, our numerical experience with various boundary conditions at $x=1$ shows that in practice the bound in (3.20) is always $O\left(K h^{m}+h^{m+1-\delta}\right)$ for $m-1 \geq 0$, so Method II is in general competitive with Method I. This improvement of (3.20) will be investigated elsewhere.

4. Numerical results. To check the sharpness of the theoretical convergence bounds in Theorems 3.3 and 3.5, we test Methods I and II on a single problem of the form (1.5) for the cases of Dirichlet and Neumann boundary conditions at $x=1$. In each numerical example one has

$$
\begin{aligned}
& u(x)=(0.3)^{-1}\left[E_{1-\delta, 1}\left(0.3 x^{1-\delta}\right)-1\right]+2 x^{2-\delta}-x^{3-2 \delta}-3 x^{3}+0.5 x^{4}, \\
& b(x)=1-0.7 \cos \left(2.3 x^{2}-x^{3}\right) \text { and } c(x) \equiv 0,
\end{aligned}
$$

with $(\alpha, \beta)=(1,0)$ or $(0,1)$. The Mittag-Leffler function $E$ used here was defined in (2.13). The function $f$ on the right-hand side of (1.5a) is specified by $L u=f$ and $\gamma$ is chosen such that (1.5b) is satisfied

Our example is constructed to have a known solution $u$ that mimics as far as possible the behaviour of a typical solution of (1.5). For one can easily check that the derivatives of our $u$ behave exactly as predicted by Corollary 2.6; furthermore, $b(x)=0.3+O\left(x^{2}\right)$ near $x=0$ and the function $\phi(x):=(0.3)^{-1}\left[E_{1-\delta, 1}\left(0.3 x^{1-\delta}\right)-1\right]$ is a solution of $-D^{2-\delta} \phi+0.3 \phi^{\prime}=0$, as can be seen using Lemma 2.1 and [3, Theorem 4.3], so $u$ has a singular component that near $x=0$ lies (almost exactly) in the null space of the differential operator as required by Remark 2.2 .

EXAMPle 4.1. Neumann condition at $x=1$, Method $I$.

Results are presented in Tables 4.1-4.6; each table is for particular choices of $m$ and the collocation parameters $\left\{c_{1}, c_{2}, \ldots, c_{m}\right\}$ in our collocation method. The convergence rates obtained agree exactly with Theorem 3.3.

EXAMPle 4.2. Neumann condition at $x=1$, Method II.

Results are presented in Tables 4.7-4.10. This is the sole example where our numerical results are better than the rates predicted by our theory: while (3.20) guarantees only $O\left(K h^{m-1}+h^{m-\delta}\right)$, the actual rates observed are $O\left(h^{m}\right)$ when $K \neq 0$ and $O\left(h^{m+1-\delta}\right)$ when $K=0$.

ExAmple 4.3. Dirichlet condition at $x=1$, Method II.

When one has a Dirichlet boundary condition at $x=1$, Method II has the same convergence bound at Method I and is moreover simpler to implement, so we do not consider Method I for this example. Tables 4.11-4.16 present the errors and rates of convergence for Method II. The convergence rates in these tables match exactly the rates predicted by Theorem 3.5. 
TABLE 4.1

Method I, case $\alpha=0, \beta=1: \max \left|\left(u-u_{h}\right)\left(x_{i}\right)\right|$ for $m=1, c_{k}=\{0\} ; K \neq 0$.

\begin{tabular}{rrrrrrrr}
\hline & $N=2^{7}$ & $N=2^{8}$ & $N=2^{9}$ & $N=2^{10}$ & $N=2^{11}$ & $N=2^{12}$ & $N=2^{13}$ \\
\hline$\delta=0.1$ & $1.92 \mathrm{e}-2$ & $9.58 \mathrm{e}-3$ & $4.78 \mathrm{e}-3$ & $2.39 \mathrm{e}-3$ & $1.19 \mathrm{e}-3$ & $5.97 \mathrm{e}-4$ & $2.98 \mathrm{e}-4$ \\
& 1.01 & 1.00 & 1.00 & 1.00 & 1.00 & 1.00 & \\
$\delta=0.3$ & $2.92 \mathrm{e}-2$ & $1.45 \mathrm{e}-2$ & $7.23 \mathrm{e}-3$ & $3.61 \mathrm{e}-3$ & $1.80 \mathrm{e}-3$ & $9.00 \mathrm{e}-4$ & $4.50 \mathrm{e}-4$ \\
& 1.01 & 1.00 & 1.00 & 1.00 & 1.00 & 1.00 & \\
$\delta=0.5$ & $5.30 \mathrm{e}-2$ & $2.61 \mathrm{e}-2$ & $1.29 \mathrm{e}-2$ & $6.39 \mathrm{e}-3$ & $3.18 \mathrm{e}-3$ & $1.58 \mathrm{e}-3$ & $7.89 \mathrm{e}-4$ \\
& 1.02 & 1.02 & 1.01 & 1.01 & 1.01 & 1.00 & \\
$\delta=0.7$ & $1.29 \mathrm{e}-1$ & $6.28 \mathrm{e}-2$ & $3.06 \mathrm{e}-2$ & $1.50 \mathrm{e}-2$ & $7.34 \mathrm{e}-3$ & $3.61 \mathrm{e}-3$ & $1.78 \mathrm{e}-3$ \\
& 1.04 & 1.04 & 1.03 & 1.03 & 1.02 & 1.02 & \\
$\delta=0.9$ & $6.29 \mathrm{e}-1$ & $3.10 \mathrm{e}-1$ & $1.53 \mathrm{e}-1$ & $7.56 \mathrm{e}-2$ & $3.72 \mathrm{e}-2$ & $1.83 \mathrm{e}-2$ & $8.97 \mathrm{e}-3$ \\
& 1.02 & 1.02 & 1.02 & 1.02 & 1.03 & 1.03 & \\
\hline
\end{tabular}

TABLE 4.2

Method I, case $\alpha=0, \beta=1: \max \left|\left(u-u_{h}\right)\left(x_{i}+c_{k} h_{i}\right)\right|$ for $m=1, c_{k}=\left\{\frac{1}{2}\right\} ; K=0$.

\begin{tabular}{rrrrrrrr}
\hline & $N=2^{7}$ & $N=2^{8}$ & $N=2^{9}$ & $N=2^{10}$ & $N=2^{11}$ & $N=2^{12}$ & $N=2^{13}$ \\
\hline$\delta=0.1$ & $7.50 \mathrm{e}-5$ & $2.12 \mathrm{e}-5$ & $5.98 \mathrm{e}-6$ & $1.68 \mathrm{e}-6$ & $4.67 \mathrm{e}-7$ & $1.30 \mathrm{e}-7$ & $3.59 \mathrm{e}-8$ \\
& 1.82 & 1.83 & 1.84 & 1.84 & 1.85 & 1.85 & \\
$\delta=0.3$ & $5.49 \mathrm{e}-4$ & $1.77 \mathrm{e}-4$ & $5.64 \mathrm{e}-5$ & $1.79 \mathrm{e}-5$ & $5.62 \mathrm{e}-6$ & $1.76 \mathrm{e}-6$ & $5.49 \mathrm{e}-7$ \\
& 1.63 & 1.65 & 1.66 & 1.67 & 1.67 & 1.68 & \\
$\delta=0.5$ & $3.03 \mathrm{e}-3$ & $1.11 \mathrm{e}-3$ & $4.00 \mathrm{e}-4$ & $1.44 \mathrm{e}-4$ & $5.14 \mathrm{e}-5$ & $1.83 \mathrm{e}-5$ & $6.51 \mathrm{e}-6$ \\
& 1.45 & 1.47 & 1.48 & 1.48 & 1.49 & 1.49 & \\
$\delta=0.7$ & $1.52 \mathrm{e}-2$ & $6.31 \mathrm{e}-3$ & $2.61 \mathrm{e}-3$ & $1.07 \mathrm{e}-3$ & $4.37 \mathrm{e}-4$ & $1.78 \mathrm{e}-4$ & $7.26 \mathrm{e}-5$ \\
& 1.26 & 1.28 & 1.28 & 1.29 & 1.29 & 1.30 & \\
$\delta=0.9$ & $9.52 \mathrm{e}-2$ & $4.37 \mathrm{e}-2$ & $2.03 \mathrm{e}-2$ & $9.47 \mathrm{e}-3$ & $4.43 \mathrm{e}-3$ & $2.07 \mathrm{e}-3$ & $9.66 \mathrm{e}-4$ \\
& 1.12 & 1.11 & 1.10 & 1.10 & 1.10 & 1.10 & \\
\hline
\end{tabular}

5. Conclusions. It was shown that a two-point boundary value problem whose highest-order derivative is a Riemann-Liouville fraction derivative (of order $2-\delta$, with $0<\delta<1$ ) could be reformulated in terms of a pair of weakly singular Volterra integral equations of the second kind. This reformulation enabled us to prove existence and uniqueness of a solution to the boundary value problem. It also led to the development of two efficient collocation methods for solving the original problem. One of these (Method II) is simpler than the other (Method I) but our error estimate for Method II is, for certain data, less good than our error estimate for Method I. Nevertheless, our numerical experience has been that both methods achieve the same rates of convergence in practice; a theoretical justification of this observation is a topic for future research.

Appendix. The piecewise polynomial collocation method. In this appendix we describe briefly the collocation method of $[1,6]$ that is used to solve Volterra integral equations of the form

$$
\mathcal{I} W=J^{1-\delta} g
$$

where $\mathcal{I}$ is defined in (3.4) and $g \in C^{q, \delta}(0,1]$ is arbitrary.

Let $N$ be a positive integer. Divide [0,1] by the mesh $0=x_{0}<x_{1}<\cdots<$ $x_{N}=1$, where $x_{i}=(i / N)^{\rho}$ for $i=0,1, \ldots, N$. The user-chosen parameter $\rho \in[1, \infty)$ determines the grading of the mesh; when $\rho=1$ the mesh is uniform. 
RIEMANN-LIOUVILLE TWO-POINT BOUNDARY VALUE PROBLEM

TABLE 4.3

Method I, case $\alpha=0, \beta=1: \max \left|\left(u-u_{h}\right)\left(x_{i}\right)\right|$ for $m=2, c_{k}=\{0,1\} ; K \neq 0$.

\begin{tabular}{rrrrrrrr}
\hline & $N=2^{6}$ & $N=2^{7}$ & $N=2^{8}$ & $N=2^{9}$ & $N=2^{10}$ & $N=2^{11}$ & $N=2^{12}$ \\
\hline$\delta=0.1$ & $8.33 \mathrm{e}-4$ & $2.08 \mathrm{e}-4$ & $5.21 \mathrm{e}-5$ & $1.30 \mathrm{e}-5$ & $3.26 \mathrm{e}-6$ & $8.14 \mathrm{e}-7$ & $2.04 \mathrm{e}-7$ \\
& 2.00 & 2.00 & 2.00 & 2.00 & 2.00 & 2.00 & \\
$\delta=0.3$ & $9.96 \mathrm{e}-4$ & $2.50 \mathrm{e}-4$ & $6.26 \mathrm{e}-5$ & $1.57 \mathrm{e}-5$ & $3.92 \mathrm{e}-6$ & $9.81 \mathrm{e}-7$ & $2.45 \mathrm{e}-7$ \\
& 1.99 & 2.00 & 2.00 & 2.00 & 2.00 & 2.00 & \\
$\delta=0.5$ & $1.09 \mathrm{e}-3$ & $2.78 \mathrm{e}-4$ & $7.02 \mathrm{e}-5$ & $1.77 \mathrm{e}-5$ & $4.44 \mathrm{e}-6$ & $1.11 \mathrm{e}-6$ & $2.79 \mathrm{e}-7$ \\
& 1.98 & 1.98 & 1.99 & 1.99 & 2.00 & 2.00 & \\
$\delta=0.7$ & $1.82 \mathrm{e}-3$ & $4.62 \mathrm{e}-4$ & $1.17 \mathrm{e}-4$ & $2.97 \mathrm{e}-5$ & $7.50 \mathrm{e}-6$ & $1.89 \mathrm{e}-6$ & $4.76 \mathrm{e}-7$ \\
& 1.98 & 1.98 & 1.98 & 1.98 & 1.99 & 1.99 & \\
$\delta=0.9$ & $2.20 \mathrm{e}-2$ & $5.75 \mathrm{e}-3$ & $1.52 \mathrm{e}-3$ & $3.99 \mathrm{e}-4$ & $1.04 \mathrm{e}-4$ & $2.72 \mathrm{e}-5$ & $7.06 \mathrm{e}-6$ \\
& 1.93 & 1.92 & 1.93 & 1.93 & 1.94 & 1.95 & \\
\hline
\end{tabular}

TABLE 4.4

Method I, case $\alpha=0, \beta=1: \max \left|\left(u-u_{h}\right)\left(x_{i}+c_{k} h_{i}\right)\right|$ for $m=2, c_{k}=\left\{0, \frac{2}{3}\right\} ; K=0$.

\begin{tabular}{rrrrrrrr}
\hline & $N=2^{6}$ & $N=2^{7}$ & $N=2^{8}$ & $N=2^{9}$ & $N=2^{10}$ & $N=2^{11}$ & $N=2^{12}$ \\
\hline$\delta=0.1$ & $6.41 \mathrm{e}-6$ & $8.80 \mathrm{e}-7$ & $1.21 \mathrm{e}-7$ & $1.65 \mathrm{e}-8$ & $2.24 \mathrm{e}-9$ & $3.05 \mathrm{e}-10$ & $4.14 \mathrm{e}-11$ \\
& 2.86 & 2.87 & 2.87 & 2.88 & 2.88 & 2.88 & \\
$\delta=0.3$ & $3.41 \mathrm{e}-5$ & $5.47 \mathrm{e}-6$ & $8.67 \mathrm{e}-7$ & $1.36 \mathrm{e}-7$ & $2.13 \mathrm{e}-8$ & $3.32 \mathrm{e}-9$ & $5.15 \mathrm{e}-10$ \\
& 2.64 & 2.66 & 2.67 & 2.68 & 2.68 & 2.69 & \\
$\delta=0.5$ & $1.11 \mathrm{e}-4$ & $2.08 \mathrm{e}-5$ & $3.83 \mathrm{e}-6$ & $6.96 \mathrm{e}-7$ & $1.25 \mathrm{e}-7$ & $2.24 \mathrm{e}-8$ & $4.00 \mathrm{e}-9$ \\
& 2.42 & 2.44 & 2.46 & 2.47 & 2.48 & 2.49 & \\
$\delta=0.7$ & $2.11 \mathrm{e}-4$ & $3.30 \mathrm{e}-5$ & $5.34 \mathrm{e}-6$ & $9.11 \mathrm{e}-7$ & $1.64 \mathrm{e}-7$ & $3.08 \mathrm{e}-8$ & $5.97 \mathrm{e}-9$ \\
& 2.68 & 2.63 & 2.55 & 2.48 & 2.41 & 2.37 & \\
$\delta=0.9$ & $1.25 \mathrm{e}-2$ & $2.83 \mathrm{e}-3$ & $6.77 \mathrm{e}-4$ & $1.65 \mathrm{e}-4$ & $4.01 \mathrm{e}-5$ & $9.72 \mathrm{e}-6$ & $2.34 \mathrm{e}-6$ \\
& 2.14 & 2.06 & 2.04 & 2.04 & 2.05 & 2.05 & \\
\hline
\end{tabular}

Set $h_{i}=x_{i+1}-x_{i}$ for $i=1,2, \ldots, N$. Set $h=\max h_{i}$.

Let $m$ be a user-chosen positive integer. When solving (A.1) numerically, our computed solution $W_{h}$ will lie in the space

$$
S_{m-1}^{-1}:=\left\{v:\left.v\right|_{\left(x_{i}, x_{i+1}\right)} \in \pi_{m-1}, i=0,1, \ldots, N-1\right\}
$$

comprising piecewise polynomials of degree at most $m-1$ that may be discontinuous at interior mesh points $x_{i}$. The set of collocation points in each mesh interval $\left[x_{i}, x_{i+1}\right]$ is

$$
X_{h}:=\left\{x_{i}+c_{j} h_{i}: 0 \leq c_{1}<c_{2}<\cdots<c_{m} \leq 1, i=0,1, \ldots, N-1\right\}
$$

where the collocation parameters $\left\{c_{j}\right\}$ are chosen by the user. If $c_{1}=0$ and $c_{m}=1$, then $\phi_{h}$ will lie in the space $S_{m-1}^{-1} \cap C[0,1]=: S_{m-1}^{0}$, and (to make the number of equations equal to the number of unknowns) we require $W_{h}$ to satisfy the initial condition $W_{h}(0)=0$ when solving (A.1) because $W(0)=0$.

The collocation solution $W_{h} \in S_{m-1}^{-1}$ of (A.1) is defined by

$$
W_{h}(x)-J_{h}^{1-\delta}\left(b W_{h}\right)(x)-J_{h}^{1-\delta}\left(\left(b^{\prime}+c\right) \int_{t=0}^{.} W_{h}\right)(x)=J_{h}^{1-\delta} g(x)
$$

for all $x \in X_{h} \cup\{1\}$. Here the notation $J_{h}^{1-\delta}$ indicates that to evaluate each integral $J^{1-\delta}(\cdot)$, weighted product quadrature is used with the collocation points as nodes. 
TABLE 4.5

Method I, case $\alpha=0, \beta=1: \max \left|\left(u-u_{h}\right)\left(x_{i}+c_{k} h_{i}\right)\right|$ for $m=3, c_{k}=\left\{0, \frac{1}{3}, 1\right\} ; K \neq 0$.

\begin{tabular}{rrrrrrr}
\hline & $N=2^{6}$ & $N=2^{7}$ & $N=2^{8}$ & $N=2^{9}$ & $N=2^{10}$ & $N=2^{11}$ \\
\hline$\delta=0.1$ & $2.09 \mathrm{e}-6$ & $2.61 \mathrm{e}-7$ & $3.25 \mathrm{e}-8$ & $4.06 \mathrm{e}-9$ & $5.08 \mathrm{e}-10$ & $6.35 \mathrm{e}-11$ \\
& 3.00 & 3.00 & 3.00 & 3.00 & 3.00 & \\
$\delta=0.3$ & $2.37 \mathrm{e}-6$ & $2.94 \mathrm{e}-7$ & $3.67 \mathrm{e}-8$ & $4.57 \mathrm{e}-9$ & $5.70 \mathrm{e}-10$ & $7.11 \mathrm{e}-11$ \\
& 3.01 & 3.01 & 3.00 & 3.00 & 3.00 & \\
$\delta=0.5$ & $1.42 \mathrm{e}-5$ & $1.80 \mathrm{e}-6$ & $2.27 \mathrm{e}-7$ & $2.86 \mathrm{e}-8$ & $3.58 \mathrm{e}-9$ & $4.48 \mathrm{e}-10$ \\
& 2.98 & 2.99 & 2.99 & 3.00 & 3.00 & \\
$\delta=0.7$ & $9.11 \mathrm{e}-5$ & $1.18 \mathrm{e}-5$ & $1.54 \mathrm{e}-6$ & $2.00 \mathrm{e}-7$ & $2.56 \mathrm{e}-8$ & $3.26 \mathrm{e}-9$ \\
& 2.94 & 2.94 & 2.95 & 2.96 & 2.97 & \\
$\delta=0.9$ & $1.63 \mathrm{e}-3$ & $2.25 \mathrm{e}-4$ & $2.93 \mathrm{e}-5$ & $3.90 \mathrm{e}-6$ & $5.24 \mathrm{e}-7$ & $6.99 \mathrm{e}-8$ \\
& 2.86 & 2.94 & 2.91 & 2.90 & 2.91 & \\
\hline
\end{tabular}

TABLE 4.6

Method I, case $\alpha=0, \beta=1: \max \left|\left(u-u_{h}\right)\left(x_{i}+c_{k} h_{i}\right)\right|$ for $m=3, c_{k}=\left\{0, \frac{1}{2}, 1\right\} ; K=0$.

\begin{tabular}{rrrrrrr}
\hline & $N=2^{6}$ & $N=2^{7}$ & $N=2^{8}$ & $N=2^{9}$ & $N=2^{10}$ & $N=2^{11}$ \\
\hline$\delta=0.1$ & $2.24 \mathrm{e}-7$ & $1.46 \mathrm{e}-8$ & $9.41 \mathrm{e}-10$ & $6.00 \mathrm{e}-11$ & $3.80 \mathrm{e}-12$ & $2.42 \mathrm{e}-13$ \\
& 3.93 & 3.96 & 3.97 & 3.98 & 3.97 & \\
$\delta=0.3$ & $8.47 \mathrm{e}-7$ & $6.21 \mathrm{e}-8$ & $4.49 \mathrm{e}-9$ & $3.23 \mathrm{e}-10$ & $2.34 \mathrm{e}-11$ & $1.71 \mathrm{e}-12$ \\
& 3.77 & 3.79 & 3.79 & 3.79 & 3.78 & \\
$\delta=0.5$ & $4.53 \mathrm{e}-6$ & $4.02 \mathrm{e}-7$ & $3.50 \mathrm{e}-8$ & $3.04 \mathrm{e}-9$ & $2.64 \mathrm{e}-10$ & $2.30 \mathrm{e}-11$ \\
& 3.49 & 3.52 & 3.53 & 3.52 & 3.52 & \\
$\delta=0.7$ & $3.23 \mathrm{e}-5$ & $3.45 \mathrm{e}-6$ & $3.54 \mathrm{e}-7$ & $3.57 \mathrm{e}-8$ & $3.58 \mathrm{e}-9$ & $3.59 \mathrm{e}-10$ \\
& 3.23 & 3.28 & 3.31 & 3.32 & 3.32 & \\
$\delta=0.9$ & $7.00 \mathrm{e}-4$ & $9.20 \mathrm{e}-5$ & $1.10 \mathrm{e}-5$ & $1.25 \mathrm{e}-6$ & $1.40 \mathrm{e}-7$ & $1.56 \mathrm{e}-8$ \\
& 2.93 & 3.07 & 3.13 & 3.16 & 3.17 & \\
\hline
\end{tabular}

That is, on each mesh interval $\left[x_{i-1}, x_{i}\right]$ the function that multiplies $(x-t)^{-\delta}$ in each expression $J^{1-\delta}(\cdots)(x)$ is replaced by a polynomial of degree $m-1$ that interpolates to this function at the collocation points $x_{i-1}+c_{j} h_{i}, j=1,2, \ldots, m$, then the resulting integrals are evaluated exactly; this procedure is described fully in $[1, \S 6.2 .2]$. By [1, Theorem 6.2.2] one then has $W_{h}$ well defined for all sufficiently small $h$.

Taking $\rho \geq m /(1-\delta)$ to obtain the optimal order of convergence in our method, by $[6$, Corollary 3.1$]$ one obtains

$$
\left\|W-W_{h}\right\| \leq C h^{m}
$$

for some constant $C$. Note that this corollary takes into account the effect of the product quadrature described above.

The iterated collocation solution $W_{h}^{i t}$ is then defined [1, Section 6.2.1] by

$$
W_{h}^{i t}(x)=J_{h}^{1-\delta}\left(b W_{h}\right)(x)+J_{h}^{1-\delta}\left(\left(b^{\prime}+c\right) \int_{0}^{(\cdot)} W_{h}\right)(x)+J_{h}^{1-\delta} g(x)
$$

for $x \in[0,1]$. Note that $W_{h}^{i t}(x)=W_{h}(x)$ for $x \in X_{h} \cup\{1\}$. Set

$$
K=\left|\int_{0}^{1} \prod_{j=1}^{m}\left(s-c_{j}\right) d s\right|
$$


TABLE 4.7

Method II, case $\alpha=0, \beta=1: \max \left|\left(u-u_{h}\right)\left(x_{i}\right)\right|$ for $m=1, c_{k}=\{0\}$, 3-point backward differencing using $\left\{x_{N-2}, x_{N-1}, x_{N}\right\}$ (NOTE: 2-point backward differencing produces similar rates of convergence, but somewhat larger errors); $K \neq 0$.

\begin{tabular}{rrrrrrrr}
\hline & $N=2^{7}$ & $N=2^{8}$ & $N=2^{9}$ & $N=2^{10}$ & $N=2^{11}$ & $N=2^{12}$ & $N=2^{13}$ \\
\hline$\delta=0.1$ & $2.57 \mathrm{e}-2$ & $1.28 \mathrm{e}-2$ & $6.39 \mathrm{e}-3$ & $3.19 \mathrm{e}-3$ & $1.60 \mathrm{e}-3$ & $7.98 \mathrm{e}-4$ & $3.99 \mathrm{e}-4$ \\
& 1.01 & 1.00 & 1.00 & 1.00 & 1.00 & 1.00 & \\
$\delta=0.3$ & $4.06 \mathrm{e}-2$ & $2.01 \mathrm{e}-2$ & $1.00 \mathrm{e}-2$ & $4.99 \mathrm{e}-3$ & $2.49 \mathrm{e}-3$ & $1.25 \mathrm{e}-3$ & $6.22 \mathrm{e}-4$ \\
& 1.01 & 1.01 & 1.00 & 1.00 & 1.00 & 1.00 & \\
$\delta=0.5$ & $7.48 \mathrm{e}-2$ & $3.66 \mathrm{e}-2$ & $1.80 \mathrm{e}-2$ & $8.91 \mathrm{e}-3$ & $4.42 \mathrm{e}-3$ & $2.20 \mathrm{e}-3$ & $1.09 \mathrm{e}-3$ \\
& 1.03 & 1.02 & 1.02 & 1.01 & 1.01 & 1.01 & \\
$\delta=0.7$ & $1.85 \mathrm{e}-1$ & $8.94 \mathrm{e}-2$ & $4.32 \mathrm{e}-2$ & $2.10 \mathrm{e}-2$ & $1.03 \mathrm{e}-2$ & $5.02 \mathrm{e}-3$ & $2.47 \mathrm{e}-3$ \\
& 1.05 & 1.05 & 1.04 & 1.04 & 1.03 & 1.02 & \\
$\delta=0.9$ & $9.18 \mathrm{e}-1$ & $4.54 \mathrm{e}-1$ & $2.25 \mathrm{e}-1$ & $1.11 \mathrm{e}-1$ & $5.46 \mathrm{e}-2$ & $2.68 \mathrm{e}-2$ & $1.31 \mathrm{e}-2$ \\
& 1.02 & 1.01 & 1.02 & 1.02 & 1.03 & 1.03 & \\
\hline
\end{tabular}

TABLE 4.8

Method II, case $\alpha=0, \beta=1: \max \left|\left(u-u_{h}\right)\left(x_{i}+c_{k} h_{i}\right)\right|$ for $m=1, c_{k}=\left\{\frac{1}{2}\right\}$, 3-point backward differencing using $\left\{x_{N-2}, x_{N-1}, x_{N}\right\} ; K=0$.

\begin{tabular}{rrrrrrrr}
\hline & $N=2^{7}$ & $N=2^{8}$ & $N=2^{9}$ & $N=2^{10}$ & $N=2^{11}$ & $N=2^{12}$ & $N=2^{13}$ \\
\hline$\delta=0.1$ & $3.06 \mathrm{e}-4$ & $7.93 \mathrm{e}-5$ & $2.06 \mathrm{e}-5$ & $5.35 \mathrm{e}-6$ & $1.39 \mathrm{e}-6$ & $3.62 \mathrm{e}-7$ & $9.45 \mathrm{e}-8$ \\
& 1.95 & 1.95 & 1.94 & 1.94 & 1.94 & 1.94 & \\
$\delta=0.3$ & $1.09 \mathrm{e}-3$ & $3.29 \mathrm{e}-4$ & $9.94 \mathrm{e}-5$ & $3.01 \mathrm{e}-5$ & $9.13 \mathrm{e}-6$ & $2.78 \mathrm{e}-6$ & $8.47 \mathrm{e}-7$ \\
& 1.73 & 1.73 & 1.72 & 1.72 & 1.72 & 1.71 & \\
$\delta=0.5$ & $5.17 \mathrm{e}-3$ & $1.83 \mathrm{e}-3$ & $6.49 \mathrm{e}-4$ & $2.29 \mathrm{e}-4$ & $8.11 \mathrm{e}-5$ & $2.87 \mathrm{e}-5$ & $1.01 \mathrm{e}-5$ \\
& 1.50 & 1.50 & 1.50 & 1.50 & 1.50 & 1.50 & \\
$\delta=0.7$ & $2.46 \mathrm{e}-2$ & $1.01 \mathrm{e}-2$ & $4.15 \mathrm{e}-3$ & $1.69 \mathrm{e}-3$ & $6.91 \mathrm{e}-4$ & $2.81 \mathrm{e}-4$ & $1.14 \mathrm{e}-4$ \\
& 1.28 & 1.29 & 1.29 & 1.30 & 1.30 & 1.30 & \\
$\delta=0.9$ & $1.51 \mathrm{e}-1$ & $6.74 \mathrm{e}-2$ & $3.12 \mathrm{e}-2$ & $1.45 \mathrm{e}-2$ & $6.80 \mathrm{e}-3$ & $3.18 \mathrm{e}-3$ & $1.48 \mathrm{e}-3$ \\
& 1.16 & 1.11 & 1.10 & 1.10 & 1.10 & 1.10
\end{tabular}

One has

$$
\left\|W-W_{h}^{i t}\right\| \leq C\left(K h^{m}+h^{m+1-\delta}\right)
$$

for some constant $C$; to see this, subtract (A.6) from (A.1) then invoke (A.5) when $K \neq 0$ and [1, Theorem 6.2.13] when $K=0$.

\section{REFERENCES}

[1] Hermann Brunner, Collocation methods for Volterra integral and related functional differential equations, vol. 15 of Cambridge Monographs on Applied and Computational Mathematics, Cambridge University Press, Cambridge, 2004.

[2] Hermann Brunner, Arvet Pedas, and Gennadi Vainikko, Piecewise polynomial collocation methods for linear Volterra integro-differential equations with weakly singular kernels, SIAM J. Numer. Anal., 39 (2001), pp. 957-982 (electronic).

[3] Kai Diethelm, The analysis of fractional differential equations, vol. 2004 of Lecture Notes in Mathematics, Springer-Verlag, Berlin, 2010. An application-oriented exposition using differential operators of Caputo type.

[4] John R. Graef, Linguu Kong, and Min Wang, A Chebyshev spectral method for solving Riemann-Liouville fractional boundary value problems, Appl. Math. Comput., 241 (2014), pp. $140-150$. 
TABLE 4.9

Method II, case $\alpha=0, \beta=1: \max \left|\left(u-u_{h}\right)\left(x_{i}\right)\right|$ for $m=2, c_{k}=\{0,1\}$, 4-point backward differencing using $\left\{x_{N-3}, x_{N-2}, x_{N-1}, x_{N}\right\} ; K \neq 0$.

\begin{tabular}{lrrrrrrr}
\hline & $N=2^{6}$ & $N=2^{7}$ & $N=2^{8}$ & $N=2^{9}$ & $N=2^{10}$ & $N=2^{11}$ & $N=2^{12}$ \\
\hline$\delta=0.1$ & $2.22 \mathrm{e}-4$ & $6.97 \mathrm{e}-5$ & $1.94 \mathrm{e}-5$ & $5.14 \mathrm{e}-6$ & $1.32 \mathrm{e}-6$ & $3.35 \mathrm{e}-7$ & $8.43 \mathrm{e}-8$ \\
& 1.67 & 1.84 & 1.92 & 1.96 & 1.98 & 1.99 & \\
$\delta=0.3$ & $5.80 \mathrm{e}-4$ & $1.84 \mathrm{e}-4$ & $5.18 \mathrm{e}-5$ & $1.37 \mathrm{e}-5$ & $3.54 \mathrm{e}-6$ & $8.99 \mathrm{e}-7$ & $2.27 \mathrm{e}-7$ \\
& 1.65 & 1.83 & 1.91 & 1.96 & 1.98 & 1.99 & \\
$\delta=0.5$ & $1.61 \mathrm{e}-3$ & $5.42 \mathrm{e}-4$ & $1.57 \mathrm{e}-4$ & $4.23 \mathrm{e}-5$ & $1.10 \mathrm{e}-5$ & $2.83 \mathrm{e}-6$ & $7.16 \mathrm{e}-7$ \\
& 1.57 & 1.79 & 1.89 & 1.94 & 1.97 & 1.98 & \\
$\delta=0.7$ & $4.23 \mathrm{e}-3$ & $1.72 \mathrm{e}-3$ & $5.47 \mathrm{e}-4$ & $1.57 \mathrm{e}-4$ & $4.26 \mathrm{e}-5$ & $1.12 \mathrm{e}-5$ & $2.90 \mathrm{e}-6$ \\
& 1.30 & 1.65 & 1.80 & 1.88 & 1.92 & 1.95 & \\
$\delta=0.9$ & $1.17 \mathrm{e}-1$ & $1.12 \mathrm{e}-2$ & $2.05 \mathrm{e}-3$ & $7.82 \mathrm{e}-4$ & $2.51 \mathrm{e}-4$ & $7.45 \mathrm{e}-5$ & $2.10 \mathrm{e}-5$ \\
& 3.39 & 2.45 & 1.39 & 1.64 & 1.75 & 1.83 & \\
\hline
\end{tabular}

TABLE 4.10

Method II, case $\alpha=0, \beta=1: \max \left|\left(u-u_{h}\right)\left(x_{i}+c_{k} h_{i}\right)\right|$ for $m=2, c_{k}=\left\{0, \frac{2}{3}\right\}$, 4-point backward differencing using $\left\{x_{N-3}, x_{N-2}, x_{N-1}, x_{N}\right\} ; K=0$.

\begin{tabular}{lrrrrrrr}
\hline & $N=2^{6}$ & $N=2^{7}$ & $N=2^{8}$ & $N=2^{9}$ & $N=2^{10}$ & $N=2^{11}$ & $N=2^{12}$ \\
\hline$\delta=0.1$ & $2.50 \mathrm{e}-4$ & $3.15 \mathrm{e}-5$ & $3.96 \mathrm{e}-6$ & $4.97 \mathrm{e}-7$ & $6.24 \mathrm{e}-8$ & $7.85 \mathrm{e}-9$ & $9.88 \mathrm{e}-10$ \\
& 2.99 & 2.99 & 2.99 & 2.99 & 2.99 & 2.99 & \\
$\delta=0.3$ & $6.88 \mathrm{e}-4$ & $9.03 \mathrm{e}-5$ & $1.18 \mathrm{e}-5$ & $1.55 \mathrm{e}-6$ & $2.05 \mathrm{e}-7$ & $2.74 \mathrm{e}-8$ & $3.68 \mathrm{e}-9$ \\
& 2.93 & 2.93 & 2.93 & 2.92 & 2.91 & 2.89 & \\
$\delta=0.5$ & $2.59 \mathrm{e}-3$ & $3.79 \mathrm{e}-4$ & $5.60 \mathrm{e}-5$ & $8.46 \mathrm{e}-6$ & $1.31 \mathrm{e}-6$ & $2.09 \mathrm{e}-7$ & $3.39 \mathrm{e}-8$ \\
& 2.77 & 2.76 & 2.73 & 2.69 & 2.65 & 2.62 & \\
$\delta=0.7$ & $1.38 \mathrm{e}-2$ & $2.36 \mathrm{e}-3$ & $4.16 \mathrm{e}-4$ & $7.62 \mathrm{e}-5$ & $1.45 \mathrm{e}-5$ & $2.84 \mathrm{e}-6$ & $5.67 \mathrm{e}-7$ \\
& 2.55 & 2.51 & 2.45 & 2.39 & 2.35 & 2.32 & \\
$\delta=0.9$ & $2.32 \mathrm{e}-1$ & $4.01 \mathrm{e}-2$ & $7.48 \mathrm{e}-3$ & $1.52 \mathrm{e}-3$ & $3.29 \mathrm{e}-4$ & $7.48 \mathrm{e}-5$ & $1.75 \mathrm{e}-5$ \\
& 2.53 & 2.42 & 2.30 & 2.21 & 2.14 & 2.10 & \\
\hline
\end{tabular}

[5] Bangti Jin, Raytcho Lazarov, Joseph Pasciak, and William Rundell, Variational formulation of problems involving fractional order differential operators, Math. Comp., (2015). (to appear).

[6] Natalia Kopteva and Martin Stynes, An efficient collocation method for a Caputo two-point boundary value problem, BIT, (2015). (to appear).

[7] Igor Podlubny, Fractional differential equations, vol. 198 of Mathematics in Science and Engineering, Academic Press Inc., San Diego, CA, 1999. An introduction to fractional derivatives, fractional differential equations, to methods of their solution and some of their applications. 
TABLE 4.11

Method II, case $\alpha=1, \beta=0: \max \left|\left(u-u_{h}\right)\left(x_{i}\right)\right|$ for $m=1, c_{k}=\{0\} ; K \neq 0$.

\begin{tabular}{rrrrrrrr}
\hline & $N=2^{7}$ & $N=2^{8}$ & $N=2^{9}$ & $N=2^{10}$ & $N=2^{11}$ & $N=2^{12}$ & $N=2^{13}$ \\
\hline$\delta=0.1$ & $1.00 \mathrm{e}-2$ & $5.02 \mathrm{e}-3$ & $2.51 \mathrm{e}-3$ & $1.26 \mathrm{e}-3$ & $6.28 \mathrm{e}-4$ & $3.14 \mathrm{e}-4$ & $1.57 \mathrm{e}-4$ \\
& 1.00 & 1.00 & 1.00 & 1.00 & 1.00 & 1.00 & \\
$\delta=0.3$ & $1.53 \mathrm{e}-2$ & $7.63 \mathrm{e}-3$ & $3.80 \mathrm{e}-3$ & $1.90 \mathrm{e}-3$ & $9.48 \mathrm{e}-4$ & $4.74 \mathrm{e}-4$ & $2.37 \mathrm{e}-4$ \\
& 1.01 & 1.00 & 1.00 & 1.00 & 1.00 & 1.00 & \\
$\delta=0.5$ & $2.80 \mathrm{e}-2$ & $1.38 \mathrm{e}-2$ & $6.80 \mathrm{e}-3$ & $3.36 \mathrm{e}-3$ & $1.67 \mathrm{e}-3$ & $8.29 \mathrm{e}-4$ & $4.13 \mathrm{e}-4$ \\
& 1.02 & 1.02 & 1.02 & 1.01 & 1.01 & 1.01 & \\
$\delta=0.7$ & $7.12 \mathrm{e}-2$ & $3.49 \mathrm{e}-2$ & $1.70 \mathrm{e}-2$ & $8.29 \mathrm{e}-3$ & $4.05 \mathrm{e}-3$ & $1.98 \mathrm{e}-3$ & $9.75 \mathrm{e}-4$ \\
& 1.03 & 1.04 & 1.04 & 1.03 & 1.03 & 1.03 & \\
$\delta=0.9$ & $3.47 \mathrm{e}-1$ & $1.88 \mathrm{e}-1$ & $9.76 \mathrm{e}-2$ & $4.94 \mathrm{e}-2$ & $2.46 \mathrm{e}-2$ & $1.21 \mathrm{e}-2$ & $5.96 \mathrm{e}-3$ \\
& 0.89 & 0.94 & 0.98 & 1.01 & 1.0 & 1.03 & \\
\hline
\end{tabular}

TABLE 4.12

Method II, case $\alpha=1, \beta=0: \max \left|\left(u-u_{h}\right)\left(x_{i}+c_{k} h_{i}\right)\right|$ for $m=1, c_{k}=\left\{\frac{1}{2}\right\} ; K=0$.

\begin{tabular}{lrrrrrrr}
\hline & $N=2^{7}$ & $N=2^{8}$ & $N=2^{9}$ & $N=2^{10}$ & $N=2^{11}$ & $N=2^{12}$ & $N=2^{13}$ \\
\hline$\delta=0.1$ & $2.33 \mathrm{e}-4$ & $6.26 \mathrm{e}-5$ & $1.68 \mathrm{e}-5$ & $4.51 \mathrm{e}-6$ & $1.21 \mathrm{e}-6$ & $3.24 \mathrm{e}-7$ & $8.68 \mathrm{e}-8$ \\
& 1.89 & 1.90 & 1.90 & 1.90 & 1.90 & 1.90 & \\
$\delta=0.3$ & $1.14 \mathrm{e}-3$ & $3.53 \mathrm{e}-4$ & $1.09 \mathrm{e}-4$ & $3.36 \mathrm{e}-5$ & $1.03 \mathrm{e}-5$ & $3.18 \mathrm{e}-6$ & $9.80 \mathrm{e}-7$ \\
& 1.69 & 1.70 & 1.70 & 1.70 & 1.70 & 1.70 & \\
$\delta=0.5$ & $5.35 \mathrm{e}-3$ & $1.91 \mathrm{e}-3$ & $6.79 \mathrm{e}-4$ & $2.41 \mathrm{e}-4$ & $8.53 \mathrm{e}-5$ & $3.02 \mathrm{e}-5$ & $1.07 \mathrm{e}-5$ \\
& 1.48 & 1.49 & 1.50 & 1.50 & 1.50 & 1.50 & \\
$\delta=0.7$ & $2.55 \mathrm{e}-2$ & $1.06 \mathrm{e}-2$ & $4.33 \mathrm{e}-3$ & $1.77 \mathrm{e}-3$ & $7.21 \mathrm{e}-4$ & $2.93 \mathrm{e}-4$ & $1.19 \mathrm{e}-4$ \\
& 1.27 & 1.29 & 1.29 & 1.30 & 1.30 & 1.30 & \\
$\delta=0.9$ & $1.70 \mathrm{e}-1$ & $8.27 \mathrm{e}-2$ & $3.95 \mathrm{e}-2$ & $1.87 \mathrm{e}-2$ & $8.77 \mathrm{e}-3$ & $4.11 \mathrm{e}-3$ & $1.92 \mathrm{e}-3$ \\
& 1.04 & 1.07 & 1.08 & 1.09 & 1.09 & 1.10 & \\
\hline
\end{tabular}

TABLE 4.13

Method II, case $\alpha=1, \beta=0: \max \left|\left(u-u_{h}\right)\left(x_{i}\right)\right|$ for $m=2, c_{k}=\{0,1\} ; K \neq 0$.

\begin{tabular}{rrrrrrrr}
\hline & $N=2^{6}$ & $N=2^{7}$ & $N=2^{8}$ & $N=2^{9}$ & $N=2^{10}$ & $N=2^{11}$ & $N=2^{12}$ \\
\hline$\delta=0.1$ & $1.82 \mathrm{e}-4$ & $4.54 \mathrm{e}-5$ & $1.13 \mathrm{e}-5$ & $2.84 \mathrm{e}-6$ & $7.09 \mathrm{e}-7$ & $1.77 \mathrm{e}-7$ & $4.43 \mathrm{e}-8$ \\
& 2.00 & 2.00 & 2.00 & 2.00 & 2.00 & 2.00 & \\
$\delta=0.3$ & $4.42 \mathrm{e}-4$ & $1.11 \mathrm{e}-4$ & $2.79 \mathrm{e}-5$ & $6.98 \mathrm{e}-6$ & $1.75 \mathrm{e}-6$ & $4.37 \mathrm{e}-7$ & $1.09 \mathrm{e}-7$ \\
& 1.99 & 2.00 & 2.00 & 2.00 & 2.00 & 2.00 & \\
$\delta=0.5$ & $1.24 \mathrm{e}-3$ & $3.17 \mathrm{e}-4$ & $8.07 \mathrm{e}-5$ & $2.04 \mathrm{e}-5$ & $5.15 \mathrm{e}-6$ & $1.30 \mathrm{e}-6$ & $3.25 \mathrm{e}-7$ \\
& 1.96 & 1.97 & 1.98 & 1.99 & 1.99 & 1.99 & \\
$\delta=0.7$ & $4.17 \mathrm{e}-3$ & $1.13 \mathrm{e}-3$ & $2.99 \mathrm{e}-4$ & $7.82 \mathrm{e}-5$ & $2.03 \mathrm{e}-5$ & $5.21 \mathrm{e}-6$ & $1.33 \mathrm{e}-6$ \\
& 1.89 & 1.91 & 1.93 & 1.95 & 1.96 & 1.97 & \\
$\delta=0.9$ & $2.07 \mathrm{e}-2$ & $6.44 \mathrm{e}-3$ & $1.90 \mathrm{e}-3$ & $5.43 \mathrm{e}-4$ & $1.51 \mathrm{e}-4$ & $4.14 \mathrm{e}-5$ & $1.12 \mathrm{e}-5$ \\
& 1.68 & 1.76 & 1.81 & 1.84 & 1.87 & 1.89 & \\
\hline
\end{tabular}


TABLE 4.14

Method II, case $\alpha=1, \beta=0: \max \left|\left(u-u_{h}\right)\left(x_{i}+c_{k} h_{i}\right)\right|$ for $m=2, c_{k}=\left\{0, \frac{2}{3}\right\} ; K=0$.

\begin{tabular}{rrrrrrrr}
\hline & $N=2^{6}$ & $N=2^{7}$ & $N=2^{8}$ & $N=2^{9}$ & $N=2^{10}$ & $N=2^{11}$ & $N=2^{12}$ \\
\hline$\delta=0.1$ & $2.14 \mathrm{e}-5$ & $2.91 \mathrm{e}-6$ & $3.92 \mathrm{e}-7$ & $5.27 \mathrm{e}-8$ & $7.07 \mathrm{e}-9$ & $9.48 \mathrm{e}-10$ & $1.27 \mathrm{e}-10$ \\
& 2.88 & 2.89 & 2.90 & 2.90 & 2.90 & 2.90 & \\
$\delta=0.3$ & $1.30 \mathrm{e}-4$ & $2.05 \mathrm{e}-5$ & $3.21 \mathrm{e}-6$ & $4.98 \mathrm{e}-7$ & $7.69 \mathrm{e}-8$ & $1.19 \mathrm{e}-8$ & $1.83 \mathrm{e}-9$ \\
& 2.66 & 2.68 & 2.69 & 2.69 & 2.70 & 2.70 & \\
$\delta=0.5$ & $8.08 \mathrm{e}-4$ & $1.50 \mathrm{e}-4$ & $2.73 \mathrm{e}-5$ & $4.92 \mathrm{e}-6$ & $8.79 \mathrm{e}-7$ & $1.57 \mathrm{e}-7$ & $2.78 \mathrm{e}-8$ \\
& 2.43 & 2.46 & 2.47 & 2.48 & 2.49 & 2.49 & \\
$\delta=0.7$ & $5.68 \mathrm{e}-3$ & $1.25 \mathrm{e}-3$ & $2.68 \mathrm{e}-4$ & $5.62 \mathrm{e}-5$ & $1.17 \mathrm{e}-5$ & $2.41 \mathrm{e}-6$ & $4.97 \mathrm{e}-7$ \\
& 2.18 & 2.23 & 2.25 & 2.27 & 2.28 & 2.28 & \\
$\delta=0.9$ & $8.38 \mathrm{e}-2$ & $2.30 \mathrm{e}-2$ & $5.92 \mathrm{e}-3$ & $1.47 \mathrm{e}-3$ & $3.56 \mathrm{e}-4$ & $8.53 \mathrm{e}-5$ & $2.03 \mathrm{e}-5$ \\
& 1.86 & 1.96 & 2.01 & 2.04 & 2.06 & 2.07 & \\
\hline
\end{tabular}

TABLE 4.15

Method II, case $\alpha=1, \beta=0: \max \left|\left(u-u_{h}\right)\left(x_{i}+c_{k} h_{i}\right)\right|$ for $m=3, c_{k}=\left\{0, \frac{1}{3}, 1\right\} ; K \neq 0$.

\begin{tabular}{rrrrrrr}
\hline & $N=2^{6}$ & $N=2^{7}$ & $N=2^{8}$ & $N=2^{9}$ & $N=2^{10}$ & $N=2^{11}$ \\
\hline$\delta=0.1$ & $1.14 \mathrm{e}-6$ & $1.39 \mathrm{e}-7$ & $1.71 \mathrm{e}-8$ & $2.11 \mathrm{e}-9$ & $2.63 \mathrm{e}-10$ & $3.28 \mathrm{e}-11$ \\
& 3.04 & 3.02 & 3.01 & 3.01 & 3.00 & \\
$\delta=0.3$ & $2.66 \mathrm{e}-6$ & $3.19 \mathrm{e}-7$ & $3.89 \mathrm{e}-8$ & $4.78 \mathrm{e}-9$ & $5.90 \mathrm{e}-10$ & $7.32 \mathrm{e}-11$ \\
& 3.06 & 3.04 & 3.02 & 3.02 & 3.01 & \\
$\delta=0.5$ & $5.59 \mathrm{e}-6$ & $6.82 \mathrm{e}-7$ & $8.45 \mathrm{e}-8$ & $1.05 \mathrm{e}-8$ & $1.30 \mathrm{e}-9$ & $1.62 \mathrm{e}-10$ \\
& 3.03 & 3.01 & 3.01 & 3.01 & 3.01 & \\
$\delta=0.7$ & $1.38 \mathrm{e}-5$ & $1.56 \mathrm{e}-6$ & $1.88 \mathrm{e}-7$ & $2.32 \mathrm{e}-8$ & $2.91 \mathrm{e}-9$ & $3.65 \mathrm{e}-10$ \\
& 3.15 & 3.05 & 3.01 & 3.00 & 2.99 & \\
$\delta=0.9$ & $1.37 \mathrm{e}-3$ & $1.91 \mathrm{e}-4$ & $2.28 \mathrm{e}-5$ & $2.60 \mathrm{e}-6$ & $2.91 \mathrm{e}-7$ & $3.38 \mathrm{e}-8$ \\
& 2.84 & 3.06 & 3.14 & 3.16 & 3.11 & \\
\hline
\end{tabular}

TABLE 4.16

Method II, case $\alpha=1, \beta=0: \max \left|\left(u-u_{h}\right)\left(x_{i}+c_{k} h_{i}\right)\right|$ for $m=3, c_{k}=\left\{0, \frac{1}{2}, 1\right\} ; K=0$.

\begin{tabular}{lrrrrrr}
\hline & $N=2^{6}$ & $N=2^{7}$ & $N=2^{8}$ & $N=2^{9}$ & $N=2^{10}$ & $N=2^{11}$ \\
\hline$\delta=0.1$ & $3.16 \mathrm{e}-7$ & $2.21 \mathrm{e}-8$ & $1.51 \mathrm{e}-9$ & $1.02 \mathrm{e}-10$ & $6.87 \mathrm{e}-12$ & $4.59 \mathrm{e}-13$ \\
& 3.84 & 3.87 & 3.89 & 3.89 & 3.90 & \\
$\delta=0.3$ & $1.33 \mathrm{e}-6$ & $1.08 \mathrm{e}-7$ & $8.59 \mathrm{e}-9$ & $6.70 \mathrm{e}-10$ & $5.19 \mathrm{e}-11$ & $4.01 \mathrm{e}-12$ \\
& 3.61 & 3.66 & 3.68 & 3.69 & 3.70 & \\
$\delta=0.5$ & $3.93 \mathrm{e}-6$ & $3.90 \mathrm{e}-7$ & $3.63 \mathrm{e}-8$ & $3.28 \mathrm{e}-9$ & $2.93 \mathrm{e}-10$ & $2.60 \mathrm{e}-11$ \\
& 3.33 & 3.42 & 3.47 & 3.49 & 3.50 & \\
$\delta=0.7$ & $1.16 \mathrm{e}-5$ & $1.03 \mathrm{e}-6$ & $9.45 \mathrm{e}-8$ & $8.86 \mathrm{e}-9$ & $8.48 \mathrm{e}-10$ & $8.26 \mathrm{e}-11$ \\
& 3.50 & 3.44 & 3.42 & 3.38 & 3.36 & \\
$\delta=0.9$ & $1.21 \mathrm{e}-3$ & $1.75 \mathrm{e}-4$ & $2.17 \mathrm{e}-5$ & $2.53 \mathrm{e}-6$ & $2.86 \mathrm{e}-7$ & $3.22 \mathrm{e}-8$ \\
& 2.79 & 3.01 & 3.10 & 3.14 & 3.15 & \\
\hline
\end{tabular}

